\title{
61. THE PROPAGATION AND ABSORPTION OF SPIRAL DENSITY WAVES
}

\author{
F. H. SHU \\ State University of New York at Stony Brook, N.Y., U.S.A.
}

\begin{abstract}
An 'anti-spiral theorem' holds with limited validity for the neutral modes of oscillation in a stellar disk - namely, whenever the effects of stellar resonances can be ignored. In the regions between Lindblad resonances, a group of spiral waves will propagate in the radial direction with the group velocity found by Toomre. This propagation occurs with the conservation of 'wave action', wave energy, and wave angular momentum.
\end{abstract}

\section{Permissibility of Modes of Spiral Form}

It is known from the work of Lynden-Bell and Ostriker (1967) that an 'anti-spiral theorem' holds for all neutral modes of oscillation associated with a differentially rotating, self-gravitating, gaseous system. However, galaxies are composed mostly of stars, and the dynamics of stars differs from that of gas (and dust) in that stars can resonate with an oscillating gravitational field without continual interruptions from collisions. These resonances arise whenever the stars feel the same phase of the perturbation gravitational field in each cycle of their peculiar motions. An exact formulation of the linearized problem, including appropriate boundary conditions, shows that an analogous 'anti-spiral theorem' holds for neutral modes in a stellar disk (modelled with infinitesimal thickness) when the effects of stellar resonances can be ignored (Shu, 1968, 1970a).

While such results pose obvious difficulties for the density wave theory, they also suggest the permissibility of spirals to depend on the prevalence of one or a combination of the following conditions:

(a) the existence of strong stellar resonances,

(b) the effects of finite amplitude,

(c) the existence of overstabilities,

(d) the driving of the disk by other agencies.

It is difficult to accept the hypothesis that resonances can by themselves generate extensive spiral patterns. Indeed, in the linear theory, the analogy with plasma physics would lead one to suspect that, except for special circumstances (possibly such as those described by Kalnajs), resonances act to absorb density waves (by mechanisms similar to Landau damping). Of course, the conversion of wave energy into energy of particle motions cannot proceed indefinitely, and non-linear effects may eventually alter these considerations.

Any expectation for waves to develop spontaneously is related to the possibility for instability. Thus, it is important that (when stellar resonances are ignored) recent investigations - summarized in the rest of this paper - reveal no short-scale instabilities in addition to the Jeans instability discussed for this geometry by Toomre (1964). 
This result leads us to consider seriously that spiral structure may be the result of forcing by a yet unspecified agency.

\section{Local Properties of Density Waves}

To investigate the properties of density waves, it is convenient to adopt the WKBJ approximation advocated by Lin (1966), but we carry the approximation one order higher than previous treatments. We treat leading and trailing spirals separately, admitting, however, the possibility that boundary conditions may ultimately require superposition.

\section{A. THE DISPERSION RELATION FOR SPIRAL DENSITY WAVES}

In the lowest order of approximation, we recover the dispersion relation of Lin and Shu (1966). Wavenumber 'information' propagates radially inside the principal range with the group velocity found by Toomre (1969). Toomre's analysis, thus, provides an important insight into the nature of 'anti-spiral theorems'. Even existing spiral waves must eventually disappear if they are neither replenished nor returned.

\section{B. THE PROPAGATION OF THE DENSITY OF WAVE ACTION}

In the next order of approximation, we obtain a relation governing the variation of amplitude with galactocentric radius (Shu, 1968, 1970b). Toomre (1969) showed that the amplitude relation of Shu can be recovered from physical considerations providing the 'density of wave action' is propagated with the group velocity. Kalnajs (private communication) further clarified this interpretation by demonstrating that the principle is equivalent to the conservation of wave energy and wave angular momentum when viewed by an observer in an inertial frame. In this approximation, then, spiral density waves of short scale show neither a tendency to be overstable nor a tendency to be damped.

Thus, the Jeans instability appears to be the only instability mechanism locally operative in the plane of a stellar disk (whose distribution function for small peculiar velocities is taken locally to be Schwarzschild's distribution). Hence, a point of great importance and some debate, is whether the Galaxy is everywhere more than marginally stable.

\section{POSSIBLE FORCING BY GRAVITATIONAL CLUMPING}

For a combined star-gas disk, one region, at least, appears unlikely to be completely stabilized. Depletion of interstellar gas by star formation is yet relatively incomplete in the outer regions of the Galaxy, and the continuous dissipation of turbulent velocities in the interstellar gas will almost certainly lead to effective values of the mean random velocities which are below that required for stability. In these regions may still occur the process of gravitational clumping of interstellar gas described by Goldreich and Lynden-Bell (1965).

Lin (1970) has proposed that gaseous condensations so produced - aided by the 
excitation of density 'wakes' in the stellar sheet (Julian and Toomre, 1966) - may serve as a source for trailing spiral waves which propagate into the interior. Even though the 'forcing' of the disk originates in the outer and more rarified regions, an impression can be made on the interior because the wave energy density tends to 'pile up' in the interior where the group velocity is small. Furthermore, because the waves are initiated as nearly co-rotating disturbances in the outer parts of the galaxy, pattern speeds characteristic of the material rotation in the outer parts would automatically result. A partial test of these ideas is possible with external galaxies since a pattern speed chosen to match the material rotation near the 'outer edge' of the observed spiral structure should yield an accurate theoretically-deduced spiral pattern for the interior.

\section{References}

Goldreich, P. and Lynden-Bell, D.: 1965, Monthly Notices Roy. Astron. Soc. 130, 125.

Julian, W. H. and Toomre, A.: 1966, Astrophys. J. 146, 810.

Lin, C. C.: 1966, SIAM J. Appl. Math 14, 876.

Lin, C. C.: 1970 IAU Symposium No. 38, p. 377.

Lin, C. C. and Shu, F. H.: 1966, Proc. Nat. Acad. Sci. U.S. A. 55, 229.

Lynden-Bell, D. and Ostriker, J. D.: 1967, Monthly Notices Roy. Astron. Soc. 136, 293.

Shu, F. H.: 1968, Ph.D. Thesis, Harvard University.

Shu, F. H.: 1970a, Astrophys. J. 160, in press.

Shu, F. H.: 1970b, Astrophys. J. 160, in press.

Toomre, A.: 1964, Astrophys. J. 139, 1217.

Toomre, A.: 1969, Astrophys. J. 158, 899. 